\title{
Reappraisal of birdshot retinochoroiditis (BRC): a global approach
}

\author{
Marina Papadia • Carl P. Herbort
}

Received: 30 July 2012 /Revised: 25 October 2012 / Accepted: 30 October 2012 /Published online: 18 November 2012

(C) Springer-Verlag Berlin Heidelberg 2012

\begin{abstract}
Background This study aimed to readjust the appraisal of birdshot retinochoroiditis (BRC) in light of a global approach, including the full array of investigational procedures.

Patients and methods This retrospective study reviewed charts of BRC cases treated in the uveitis clinic of our center between 1995 and 2011. We identified 25 patients with BRC; of these, 19 had sufficient data for inclusion in the study. Patients were examined with a standard clinical approach for inflammatory disorders, including dual fluorescence angiography with fluorescein and indocyanine green, perimetry, and laser flare photometry, both at presentation and during follow-up. Spectral optical coherence tomography (OCT) was performed when available. Disease characteristics and evolutionary patterns were reported.

Results Human leucocyte antigen was positive for the A29 allele in all patients. The mean age at presentation was $49.6 \pm 10.0$ years, the mean diagnostic delay was $21.5 \pm$ 18 months, and the mean follow-up was $85 \pm 60$ months. Out of 19 patients, three presented with mutton-fat keratic precipitates (KPs), three had no depigmented lesions at presentation, and eight did not fulfill the recommended criterion of three depigmented peripapillar lesions. Cystoid macular edema (CMO) at entry was
\end{abstract}

M. Papadia $\cdot$ C. P. Herbort $(\bowtie)$

Inflammatory and Retinal Eye Diseases, Centre for Ophthalmic Specialised Care (COS), Rue de la Grotte 6,

1003 Lausanne, Switzerland

e-mail: carl.herb@bluewin.ch

\section{Papadia}

Eye Clinic, Department of Neurosciences, Ophthalmology and

Genetics, University of Genova, Genoa, Italy

C. P. Herbort

University of Lausanne, Lausanne, Switzerland present in $8 / 19$ cases. Perimetric anomalies were noted in all patients at presentation. In $92 \%$ of cases, fluorescein findings included disc hyperfluorescence, retinal vasculitis of large vessels, and leakage from mediumsized and small vessels. In all patients, a (pseudo)-delay was noted in the arterio-venous circulation time (mean venous dye appearance $=42.1 \pm 13.1 \mathrm{~s}$ ), which reflected massive capillary leakage. At presentation, all patients exhibited indocyanine green angiographic signs, including hypofluorescent dark dots, vessel fuzziness, and areas of diffuse late hyperfluorescence. This allowed early diagnosis in $3 / 19$ patients $(16 \%)$ without birdshot fundus lesions at presentation.

Conclusions BRC is a granulomatous uveitis, and muttonfat KPs do not exclude the disease. When BRC is suspected, indocyanine green angiography is crucial to allow early diagnosis and to monitor the evolution of choroiditis. Perimetry is an obligate investigation for diagnosis and followup. $\mathrm{CMO}$ is less frequent than stated earlier. Scores of fluorescein and indocyanine green angiographic signs indicated that choroiditis responded readily to therapy, but retinitis was relatively resistant to therapy.

Keywords Birdshot retinochoroiditis · Indocyanine green angiography $\cdot$ Choroidal granuloma

\section{Introduction}

Birdshot retinochoroiditis (BRC) is an intraocular inflammatory condition, mostly observed in Caucasian populations. BRC is linked to the presence of the tissue histocompatibility antigen HLA-A29, but without any known systemic association [1,2].

To date, the appraisal of BRC has mostly been only partial and sometimes insufficient; this has given rise to 
several misconceptions of the disease. In most chorioretinal inflammatory diseases, the inflammatory process takes place in one structure, like the choroid in Vogt-Koyanagi-Harada (VKH) disease, or the retina in toxoplasmosis. Then, it extends to neighboring structures, as the inflammation becomes more severe, such as the retina and the choroid. In BRC, the pathogenesis of inflammation is unique; it occurs independently, but simultaneously in the choroid and the retina [3]. This concept has not been brought forward clearly, despite the consequences it has on the approaches for treating the disease. This dual, independent involvement can explain why both fluorescein angiography (FA) and indocyanine green angiography (ICGA) are necessary for correct assessments of intraocular inflammatory involvement, and why the kinetics of inflammation should be followed in both compartments. Unfortunately, routine ICGA, even in conditions with known predominant choroidal involvement, is still not performed routinely. For monitoring disease progression, visual field (VF) testing is crucial in BRC. Despite the fact that this has been stressed for over 10 years [4], only more recently have studies dealt with this aspect of the disease [5, 6]. Moreover, in current, routine uveitis practice, VF testing is still not acknowledged as the most relevant functional test to monitor disease progression or response to therapy, and VF testing is rarely described in studies on BRC.

The consequence of misunderstanding BRC was illustrated in the publication of international research diagnostic criteria (IRDC) for BRC; among other inadequacies, the IRDC did not even mention VF testing or ICGA [7]. In the present study, we investigated and followed a series of 19 patients with BRC. We used a comprehensive approach, including analysis of choroidal involvement, optical coherence tomography (OCT) analysis of retinal involvement, laser flare photometric analysis of anterior chamber involvement, and visual field status. We aimed to address several misconceptions about BRC and provide a global overview of the disease.

\section{Materials and methods}

We retrospectively reviewed charts of patients examined in the uveitis clinic at the Center for Ophthalmic Specialized Care, Lausanne, Switzerland between 1995 and 2010. Patients with a BRC diagnosis were identified. Diagnostic criteria included vitreitis and retinal vasculitis in either or both eyes, visual field loss in either or both eyes, stromal choroiditis in both eyes evidenced by ICGA, and the presence of the HLA-A29 antigen. An additional, but not obligatory criterion was the presence of rice-shaped, depigmented "birdshot lesions". All patients underwent the complete workup routinely applied to patients with posterior uveitis. Routine examinations included the Snellen visual acuity test, slit-lamp examination, applanation tonometry, and funduscopy in mydriasis. In addition, at each major visit, a complete ocular examination was performed that comprised laser flare photometry, computerized VF testing, OCT (when available), and dual FA and ICGA.

The study was performed in accordance with the ethical standards laid down in the 1964 Declaration of Helsinki.

FA and ICGA were performed with a standard protocol described previously [8]. Typically, FA and ICGA were performed simultaneously. Images were acquired with a Topcon 50 IA camera (Tokyo, Japan) coupled to an ImageNet (Topcon) image digitizing system or a Heidelberg Retina Angiograph HRA 2 (Heidelberg Engineering Inc).

Autofluorescence was excluded by performing preinjection fluorescence detection with the highest flash intensity used for ICGA. At the same time, red-free posterior pole photographs were taken. Next, a bolus injection was administered of $500 \mathrm{mg}$ indocyanine green (ICG) (Cardiogreen; Peaselt, Lorei, Germany) diluted in $7.5 \mathrm{ml}$ of $0.9 \%$ normal saline. Then, ICGA photographs were taken of the posterior pole for approximately 2 to $3 \mathrm{~min}$ (early phase angiogram). ICG background fluorescence was analyzed in detail at $12 \pm 3$ min after the ICG injection (intermediate phase of angiogram) by taking photographs of the posterior pole and a minimum of eight frames of the entire periphery $\left(360^{\circ}\right)$. At the end of the intermediate phase of ICGA, FA was performed. Early FA frames of the posterior pole were taken for $2 \mathrm{~min}$. Then, between 4 and $7 \mathrm{~min}$, a $360^{\circ}$ panorama image was performed over the periphery (minimum eight frames); finally, at $10 \mathrm{~min}$, late fluorescein frames were taken of the posterior pole. Late ICG background fluorescence was analyzed at $30 \pm 4 \mathrm{~min}$ after the ICG injection (late-phase angiogram); this was performed in the same fashion as that used for the background analysis of the intermediate phase.

Laser flare assessment was performed with a Kowa FM500 (Kowa Company, Ltd., Electronics and Optics Division, Tokyo, Japan). OCT was performed with an OTI-Spectral OCT/SLO (OTI Inc., Toronto, Canada) or with a Heidelberg Spectralis OCT (Heidelberg Engineering Inc.). VF assessments were performed with the G1 program of the OCTOPUS 900 (Octopus 900, G Standard; Haag-Streit International).

The data for every patient was assessed for typical, discolored, birdshot fundus lesions, ICGA dark dots, and FA evidence of vascular disturbances. The findings were analyzed and correlated to each other, as described previously [3]. The fundus score $(0-4)$ was based on the distinctness and number of discolored lesions. The ICGA score $(0-4)$ was based on several criteria, including hypofluorescent dark dots (HDDs), choroidal vessel fuzziness, disk hyperfluorescence, and late, diffuse, choroidal hyperfluorescence. The FA score (0-4) was based on vascular leakage of small 
and large retinal vessels, disk hyperfluorescence, and macular edema. The data were scored by a blinded investigator (M.P.). Evaluations were performed at different times, including before treatment, after treatment in patients with disease that had evolved from 1 to 6 years, and after treatment in patients with disease durations over 6 years. The scores from different times were statistically compared with a Student's $t$-test. The term "at presentation" indicates when the patient was first seen in our center, not at the onset of the disease.

\section{Results}

\section{Demographics}

From 1995 to 2010, 1,268 new patients were examined at the uveitis clinic at the Center for Ophthalmic Specialized Care, Lausanne, Switzerland. Of those, 25 patients (2\%; 19 women/6 men, sex ratio 3:1) were diagnosed with BRC. Nineteen patients had sufficient data to be included in the study.

The mean age at BRC onset was $49.6 \pm 10$ years and the mean diagnostic delay from the onset of symptoms to diagnosis was $21.5 \pm 18$ months. The mean follow-up was $85 \pm 60$ months. All patients were positive for the histocompatibility antigen, HLA-A29 (100\%). The mean bestcorrected visual acuity (BCVA) at presentation was $0.84 \pm 3.4$ in the right eye (RE) and $0.74 \pm 3.8$ in the left eye (LE). At last follow-up, the mean BCVA was $0.98 \pm 0.25 \mathrm{RE}$ and $0.92 \pm 0.36 \mathrm{LE}$ ( $p$ was non-significant).

No patient presented with severe hypertension, and for security purpose five patients with introcular pressure around $20 \mathrm{mmhg}$ were given treatment with an association of timolol and dorzolamide (Cosopt $\left.{ }^{\circledR}\right)$ twice daily.

\section{Anterior segment findings}

Anterior inflammatory involvement was very scarce in BRC. In this study, the mean anterior chamber flare measured by laser flare photometry in 38 eyes at presentation with BRC was $8.85 \pm 3.36 \mathrm{ph} / \mathrm{ms}$ (range $4.7-17.1 \mathrm{ph} / \mathrm{ms}$ ); this result implied that laser flare photometry was not a useful follow-up procedure for BRC. None of the eyes exhibited iridolenticular synechiae; however, 3/19 $(15.8 \%)$ patients exhibited granulomatous keratic precipitates (KPs). Two patients exhibited a single KP in one eye, and the third patient exhibited two KPs in one eye.

\section{Posterior segment findings}

Vitreous cells were present in all 19 patients at presentation. Cystoid macular edema (CMO) was noted in 14 eyes (39\%) of eight patients (44\%) at presentation. Oval, depigmented, birdshot fundus lesions were observed in 16 patients $(84 \%)$ at presentation (Fig. 1). In three patients (16\%), absolutely no birdshot fundus lesion was detected (Fig. 2). However, among the seven patients that presented in the initial phase of the disease (with symptoms for 1 year or less), three (43\%) exhibited birdshot fundus lesions. In these three patients, the diagnosis was based on the combined observations of vitreitis, retinal vasculitis on the FA, typical HDDs on the ICGA, and a positive HLA-A29 test. Four other patients had very faint lesions, but none in the peripapillar area; therefore, those patients would not have been diagnosed with BRC based on IRDC criteria, because they did not fulfill one of the major diagnostic criteria of the IRDC (depigmented peripapillary oval lesions) [7]. Based on the IRDC criteria, a total of 7/19 (37\%) patients should not have been diagnosed with BRC. Papillitis was clinically present in 15 eyes (39\%) of nine patients (47\%).

Visual field data

Visual field defects were found in at least one eye for all patients at presentation (100\%). No common characteristic pattern could be found in the VF defects among these patients. Moreover, when two defects were observed in the same patient, the two eyes demonstrated different defect patterns. The most frequent findings were clusters of relatively moderate defects at the borders of the area explored (G1 program of the Octopus perimeter) and in the temporal hemi-field, particularly in the peripapillary area.

At presentation, severe visual field defects were found in $12 / 38$ eyes ( $32 \%$ ) of $7 / 19$ patients ( $37 \%$ ). A visual acuity of 1.0 was noted in $5 / 38$ eyes $(13 \%)$ of $3 / 19$ patients ( $16 \%$ ) due to tubular visual field impairment.

\section{Fluorescein angiography}

Among these patients with BRC, a typical feature was vasculitis in the retinal veins of the arcades, with fuzzy hyperfluorescence and profuse capillary leakage at the posterior pole and the periphery (Fig. 3). CMO was found in 14/ 38 eyes $(37 \%)$ of $8 / 19$ patients $(42 \%)$, at much less frequency than previously reported [9-11]. Astonishingly, in some eyes, despite massive capillary leakage at the posterior pole and the periphery, the macula was devoid of CMO (Fig. 3). On FA, disc hyperfluorescence was found in $35 / 38$ eyes ( $92 \%$ ) of $17 / 19$ patients $(89 \%)$. The substantial loss of fluorescein from capillaries caused a significant delay in the impregnation of the large veins; the mean venous filling time was $42.07 \pm 13.1 \mathrm{~s}$. This finding was previously described by Gass as the arterio-venous circulation delay [12]. However, that interpretation was misleading, because the arterio-venous circulation time was normal 
Fig. 1 Typical oval

depigmented birdshot fundus lesions in a right and $\mathbf{b}$ left eye
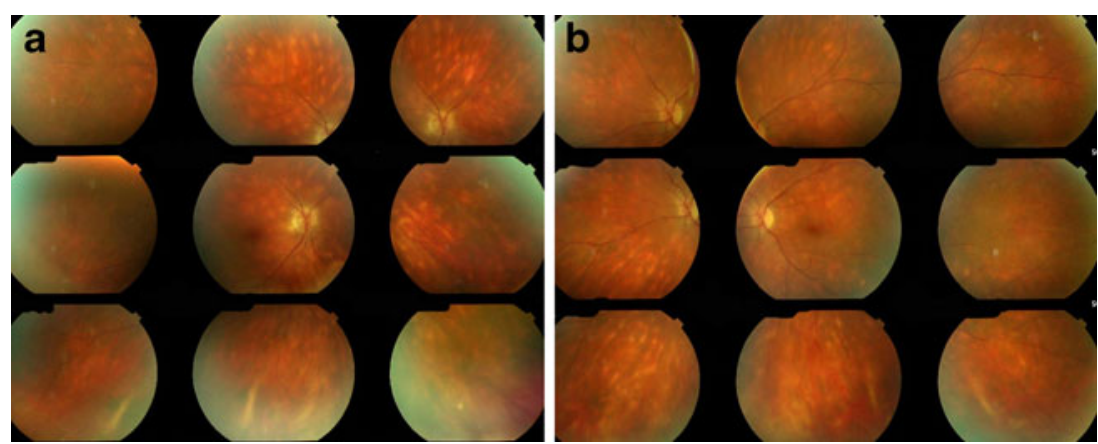

on the ICGA, around 18-22 $\mathrm{s}$ in all cases (Fig. 4). The pseudo-arterio-venous delay could be explained by the pronounced loss of fluorescein into the retina due to profuse capillary leakage; thus, reduced amounts of fluorescein reached the large veins, and this resulted in a delay in filling the large veins [13]. In contrast, ICG is a large molecular complex that does not leak from retinal capillaries; thus, it showed normal arterio-venous circulation times. a

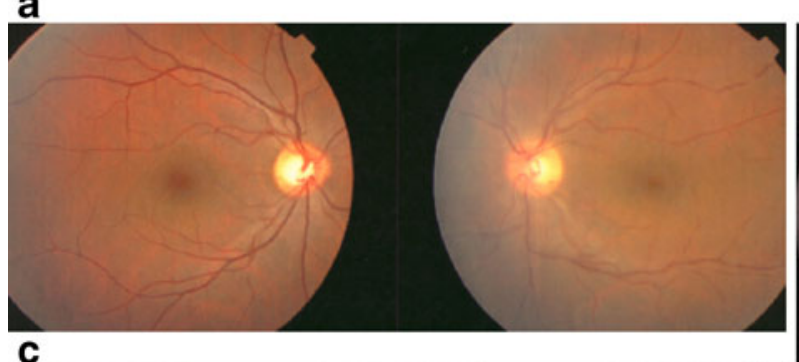

C
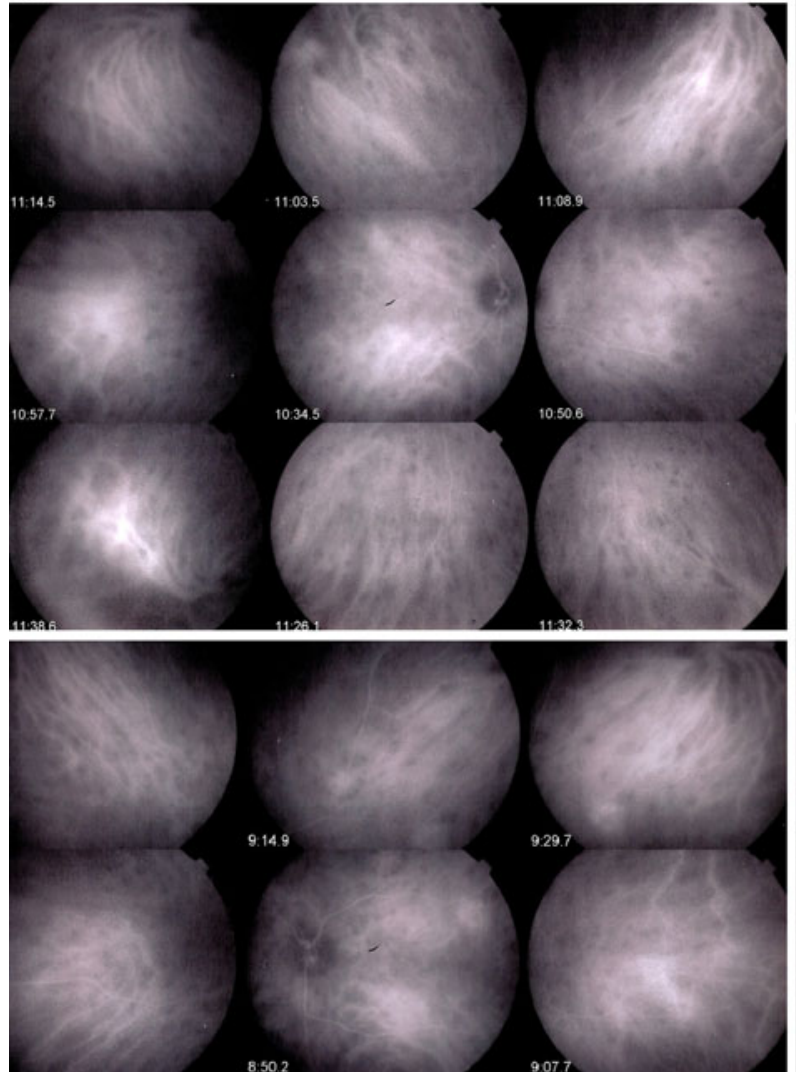

Fig. 2 Fundus images of right and left eye of a patient that complained of blurred vision in the left eye. a The fundus appeared hazy in the affected eye. No birdshot lesions were visible in either eye. b Fluorescein angiographic image shows venous sheathing compatible with BRC. c

\section{b}
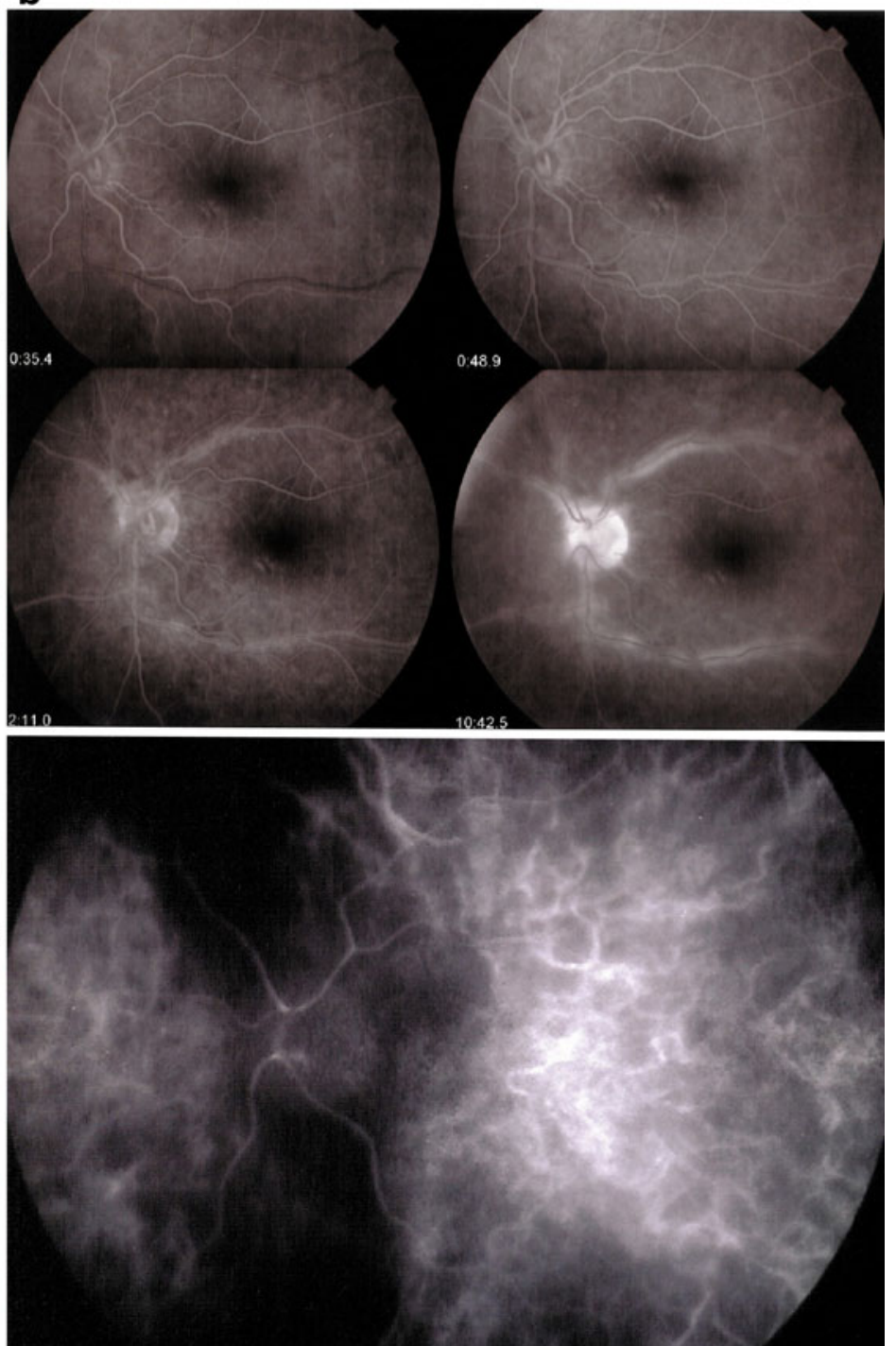

Indocyanine green angiographic image shows typical hypofluorescent dark dots (HDDs) and fuzzy choroidal vessels, in both (1) the left, symptomatic eye, and (2) the right eye. These signs strongly suggested the diagnosis of BRC, and this was confirmed by HLA-A29 positivity 
Fig. 3 Fluorescein angiographic findings in BRC. Image shows massive perivascular sheathing of arcade vessels with massive leakage from medium-sized vessels and capillary leakage. Note the $\mathrm{CMO}$ on the right side, but the absence of CMO on the left side, despite massive leakage.

Note also hyperfluorescent discs in both eyes
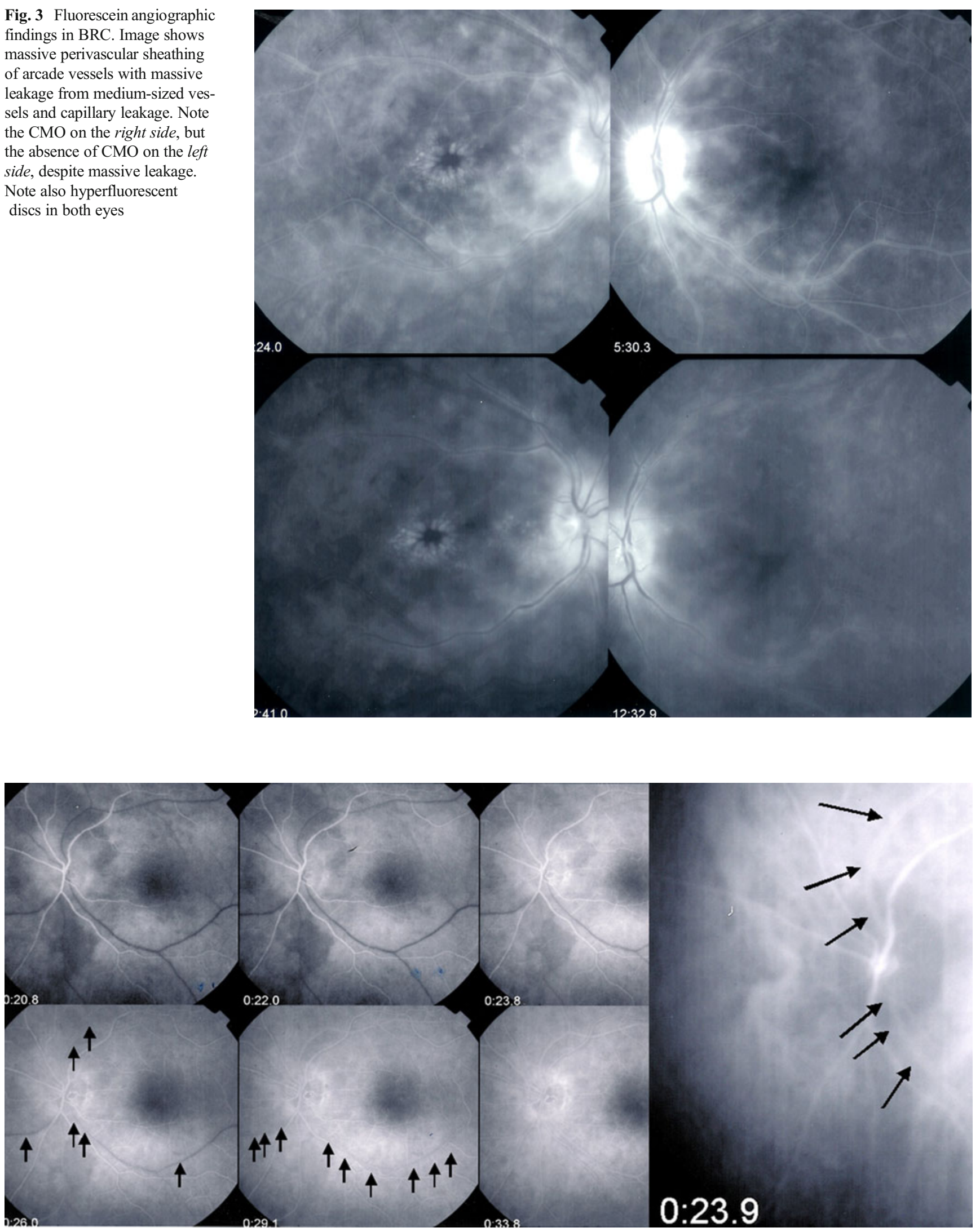

Fig. 4 Pseudo-perfusion delay in BRC. Fluorescein dye showed incomplete filling of large veins; venous filling required up to $29 \mathrm{~min}$, $1 \mathrm{~s}$. In contrast, ICGA shows complete arterio-venous filling in $23.9 \mathrm{~s}$.
Arrows outline (left frames) incomplete dye filling in FA and (right frame) fully perfused veins in ICGA 
Indocyanine green angiography (ICGA)

The most constant and consistent finding on ICGA images was the presence of evenly-sized, round-oval, hypofluorescent dark dots (HDDs), regularly distributed over the fundus in $100 \%$ of cases. In some cases $(21 \%)$, these HDDs were detected only up to the intermediate angiographic time (8-12 min), which indicated partial-thickness granulomas. The other $79 \%$ of cases showed HDDs up to the late angiographic time (26-36 min), which indicated large, fullthickness granulomas, like those observed in VKH disease [14]. In three patients, the presence of HDDs led to a diagnosis in the early stage of disease, in the absence of depigmented oval birdshot lesions (Fig. 2). All cases exhibited fuzzy, indistinct, large, choroidal vessels and late, diffuse, choroidal hyperfluorescence, but they were much less pronounced than those described in VKH disease [15]. Disc hyperfluorescence was only observed in $7 / 38$ eyes (18\%) of $4 / 19$ patients $(21 \%)$. This rare occurrence indicated much less severe, less acute choroidal inflammation compared to VKH disease [16]. All these ICGA signs decreased and/or disappeared after the introduction of inflammation suppressive therapy. Even the HDDs disappeared in most cases (57\% of HDDs disappeared on ICGA after 4 years of immunosuppressive treatment).

\section{Optical coherence tomography (OCT)}

Fourteen patients had regular OCT examinations during follow-up. Intraretinal fluid was detected in 3/4 eyes in the early stages of disease. During follow-up, the intraretinal fluid diminished, probably due to therapy. In all seven patients in the late stage of disease, the retinal thickness had decreased due to atrophy (100\%); this was concomitant with the development of thin epiretinal membranes in 13/14 eyes $(93 \%)$.

\section{Treatment}

Two out of 19 patients had only local treatment; each received two subTenon's injections in the predominantly involved eye, which stabilized the condition. They both experienced visual field recovery and visual acuity improvements. Follow-up was 12 years in one patient, but only 4 years in the other patient. This indicated that the proportion of patients with a benign evolution could be estimated as one out of ten. One patient refused treatment, and evolution was deleterious, with vision of $0.5 \mathrm{OU}$; the ICGA demonstrated extensive retinal vasculitis, $\mathrm{CMO}$, papillitis, and extensive choroidal involvement.

All the remaining patients (16 patients) received at least two inflammation suppressive treatments. Fourteen patients received prednisone, beginning with a high dose that was later tapered. In 11 out of 14 patients, systemic corticosteroid could be stopped or reduced to less than $10 \mathrm{mg} /$ day. In the other three patients, the dose was $10 \mathrm{mg} / \mathrm{day}$. Due to diabetes, two patients received periocular subtenon injections (2-3 injections per eye, given at 1 month intervals) in parallel with azathioprine in one patient and mycophenolate in the other patient. The mean duration for systemic therapy was $9.14 \pm 4.20$ years, which indicated that the disease ran a severe course in a large proportion of cases.

Table 1 provides an overview of the distribution of specific immunosuppressive treatments among the 19 patients in this study.

\section{Evolution of treated patients}

At the end of follow-up, visual acuity was $0.98 \pm 0.25$ in RE and $0.92 \pm 0.36$ in LE.

Anterior chamber inflammation remained low, with mean flare values of $9.14 \pm 4.83 \mathrm{ph} / \mathrm{ms}$ (range $3.7-18.4 \mathrm{ph} / \mathrm{ms}$ ) and the granulomatous KPs had disappeared.

Visual field was recovered in 12/18 patients after 1 year of treatment. However, 6/18 patients (33\%) (eight eyes) retained significant visual field defects, with tubular vision in three patients.

Two patients evolved towards pseudo retinitis pigmentosa fundi, which corresponded to thin, atrophic retinas on the OCT. In contrast, untreated patients exhibited extensive exudative retinitis and disc hyperfluorescence.

Angiographic evolution was characterized by a good response in the choroidal structures on the ICGA. The first sign of recovery was the disappearance of disk hyperfluorescence; then fuzzy vessels were restored, and late hyperfluorescence was reduced; finally, the HDDs disappeared. Response to therapy was less satisfactory in the retina (FA); there, vasculitis persisted along the veins of the arcades and capillary leakage was noted in nine out of 11 patients $(82 \%)$ with complete data sets. One patient showed persistence of retinal disease after 5 years of azathioprine therapy, and another patient responded well to azathioprine.

Disease evolution during treatment was analyzed with an arbitrary, masked, scoring system (0-4) [3]. This accounted

Table 1 Distribution of immunosuppressive treatments among 18 patients with $\mathrm{BRC}$; most patients required two drugs for adequate treatment

\begin{tabular}{ll}
\hline Drug & Number of patients \\
\hline Prednisone & 14 \\
Azathioprine & 10 \\
Cyclosporine & 5 \\
Anti-TNF & 2 \\
Mycophenolate mofetil & 5 \\
IV Immunoglobulines & 1 \\
\hline
\end{tabular}


well for the evolutionary pattern of increasing fundus lesions over time. Retinal disease remained persistent, but choroidal involvement responded well to treatment. However, when patients were examined early and received early treatment, the retinal disease also responded well to therapy (Table 2, Fig. 5).

\section{Discussion}

$\mathrm{BRC}$ is a rare disease, and investigations essential for diagnosis, like VF testing and ICGA, have still not been established as routine, or they are not reported. Therefore, BRC appraisal has remained incomplete, and misconceptions have found their way into ophthalmic literature.

We studied 19 patients that had received, in addition to the classical work-up, laser flare photometry, choroidal assessments, and VF testing, both at presentation and upon follow-up. The aim of this study was to rectify some of the misconceptions that currently surround BRC, and to mitigate the risk of having misconceptions perpetuated in future articles and textbooks.

Birdshot retinochoroiditis occupies a special place in intraocular inflammatory conditions, because the choroid and retina are both primary targets for inflammatory insults. Both structures are consistently but independently, involved in the inflammatory process. In contrast, two other conditions, tuberculous chorioretinitis and sarcoidosis, also involve inflammation of the retina and choroid, but these structures are affected randomly, not systematically, as in BRC $[17,18]$.

Moreover, most intraocular inflammatory conditions affect one primary target, and the neighboring structures are only secondarily involved. For example, in VKH disease, the inflammatory reaction is directed towards the choroidal melanocytes. It remains unknown why, in BRC, inflammation is generated simultaneously and systematically both in the retina and the choroid.

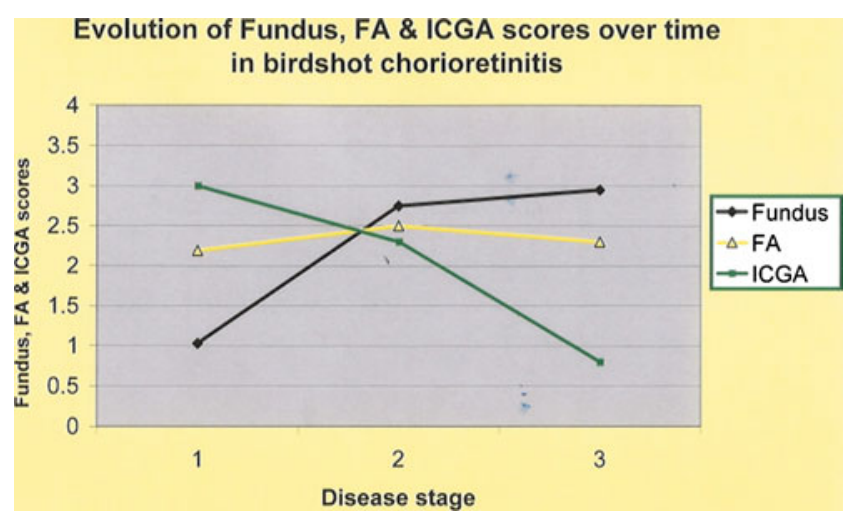

Fig. 5 Evolution of fundus lesions, FA signs, and ICGA signs over time

Until 15 years ago, only retinal involvement was detectable with angiographic examination, which did not enable global analysis of intraocular inflammation [19]. Currently, the availability of ICGA has enabled analyses and monitoring of choroidal inflammatory involvement; thus, precise appraisal of both retinal and choroidal inflammation can be performed in parallel $[8,14,17,20,21]$.

ICGA has represented a major improvement in the investigation of BRC, because it has revealed the importance of choroidal disease [17]. With ICGA, it was discovered that BRC exhibited a particular inflammatory pattern, where both the choroid and the retina were independent targets and sites of inflammation. This contrasted with the pattern of most uveitides, where inflammation typically originated in one structure or point, and inflammation in surrounding structures only occurred with disease progression [22].

The improved morphological appraisal of disease activity provided with dual fluorescein and ICGA has enabled correlations of morphological findings with functional loss. Thus, it has been possible to identify the diseased structures underlying functional loss. In fact, over 10 years ago, several groups reported extensive retinal inflammation, which was most likely the cause of visual field defects found in $100 \%$ of cases; however, to date, this finding has remained largely unrecognized [4-6, 23].
Table 2 Evolutionary profile of depigmented oval birdshot fundus lesions, fluorescein angiography (FA), and indocyanine green angiography (ICGA) over time

\begin{tabular}{llll}
\hline Subgroups & $\begin{array}{l}\text { Depigmented birdshot } \\
\text { fundus lesions (score) }\end{array}$ & FA score & ICGA score \\
\hline $\begin{array}{l}\text { Group 1: non-treated } \\
<1 \text { year duration }\end{array}$ & $1.03 \pm 0.84$ & $2.19 \pm 1.15$ & $3.0 \pm 0.65$ \\
Group 2: treated & $2.75 \pm 0.8$ & $2.5 \pm 0.97$ & \\
$1-7$ years' duration & & & $2.9 \pm 0.57$ \\
Group 3: treated & $2.95 \pm 0.79$ & $2.3 \pm 0.57$ & \\
$>7$ years' duration & $p<0.0005$ & $p=\mathrm{ns}$ & $0.8 \pm 0.27$ \\
$P: 1$ versus 2 & $p<0.002$ & $p=\mathrm{ns}$ & $p<0.05$ \\
$P: 1$ versus 3 & $p=\mathrm{ns}$ & $p=\mathrm{ns}$ & $p<0.00001$ \\
$P: 2$ versus 3 & & & $p<0.02$ \\
\hline
\end{tabular}


We previously established a scoring system to rate the severity of fundus lesions, based on FA and ICGA signs. With this scoring system, we showed that the deleterious functional evolution (visual field) observed in many BRC cases was due to persistent retinal disease [24], because the retinal system was less responsive to therapy than the choroidal system. This might be explained by differences in accessibility to the choroidal and retinal systems. The choroid is readily treated with systemic therapy through the choriocapillaris fenestrations; in contrast, the retina is more difficult to access due to blood-ocular barriers, and hence, treatment is less efficacious. On the other hand, reports are scarce on choroidal disease in BRC [3, 25-27].

The choroidal ICGA lesions (dark dots) were originally attributed to choroidal granuloma; but this hypothesis had remained conjectural until histopathological sections of the choroid from a patient with BRC had become available. The histopathology clearly showed partial-thickness granulomas in the choroidal stroma [28]. In contrast to retinal disease, choroiditis readily responds to inflammation suppressive therapy; a large proportion of HDDs regress with therapy. Nevertheless, typical, oval, depigmented birdshot lesions remain apparent, unless treatment is given early [17]. The genesis of the typical oval birdshot fundus lesion has also been conjectural, but histopathological data are available that show a granulomatous reaction against islets of stromal melanocytes. Those data have supported the hypothesis that the birdshot lesions represent areas that lack melanocytes after resolution of the granulomas [29].

Importantly, early diagnosis of BRC is only possible with ICGA, because choroidal disease is occult, and, in the absence of birdshot lesions, the typical choroidal HDDs can only be detected with ICGA.

The availability of histopathological sections has shown that BRC has granulomatous features [28]. Therefore, it is not surprising that, rarely, granulomatous KPs have been found. Although it occurs rarely, the presence of KPs should not be considered an exclusion criterion, as stated a few years ago in the article on research diagnostic criteria for birdshot chorioretinopathy [7]. The same article minimized the value of HLA-29 as a strong diagnostic element. Our study confirmed that HLA-29 is one of the most specific and sensitive diagnostic tests for BRC, when it is performed in the presence of a compatible clinical background. The frequency of HLA-A29 antigen in a Caucasoid population is $5.7 \%$; in an oriental population, the frequency is only $0.8 \%$. The relative risk for developing BRC is $224 \times$ higher in patients positive for the HLA-A29 antigen.

Another misconception generated from older publications, which should be corrected, was that BRC is a benign disease [29]. Only two of our patients exhibited a benign course. After excluding the patient that refused treatment, all our patients required at least two immunosuppressive drugs.
Moreover, our data suggested that we should be prepared for prolonged therapy. All systemically treated patients remained on low-dose maintenance therapy at the end of follow-up, and the mean treatment duration was over 9 years. In one patient, discontinuation of azathioprine therapy was attempted after 8 years, but a recurrence occurred 9 months later, with changes in vision and worsening of FA signs. Treatment was reintroduced with a short course ( 8 weeks) of oral prednisone and parallel reintroduction of azathioprine; this stabilized the disease. Our group achieved a favorable outcome with early, sustained, high-dose immunosuppressive therapy.

Considering the results from all investigations on the different compartments of the eye, including the choroid, we have gained a fairly clear, rectified depiction of BRC. We know that BRC is a granulomatous, inflammatory disease associated with HLA-A29 antigen in nearly $100 \%$ of cases. Inflammation develops independently, but simultaneously, in the retina and choroid. At the beginning, choroidal involvement is occult, and it can only be detected with ICGA. At clinical presentation, $100 \%$ of cases exhibit visual field changes linked to retinal disease. Evolution is severe in over $90 \%$ of cases, and adequate treatment requires 2-3 immunosuppressive agents for prolonged periods. Choroidal disease will readily respond to systemic inflammation suppressive therapy, as evidenced by normalization of ICGA findings. Depigmented birdshot lesions are thought to result from the destruction of melanocytic, stromal islets by the granulomatous process, and the lesions later disappear, indicated by ICGA. Retinal inflammatory involvement is less responsive to therapy, and it is responsible for deleterious evolution, which cannot be prevented in some cases. Ultimately, the retina often becomes atrophic, as evidenced by OCT, and pseudoretinitis pigmentosa may occur.

\section{References}

1. LeHoang P, Ozdemir N, Benhamou A, Tabary T, Edelson C, Betuel H, Semiglia R, Cohen JH (1992) HLA-A29.2 subtype associated with birdshot retinochoroidopathy. Am J Ophthalmol 113:32-35

2. Shah KH, Levinson RD, Yu F, Goldhardt R, Gordon LK, Gonzales CR, Heckenlively JR, Kappel PJ, Holland GN (2005) Birdshot chorioretinopathy. Surv Ophthalmol 6:519-541

3. Herbort CP, Probst K, Cimino L, Tran VT (2004) Differential inflammatory involvement in retina and choroid in birdshot chorioretinopathy. Klin Monatsbl Augenheilkd 221:351-356

4. de Courten C, Herbort CP (1998) Potential role of computerized visual field testing for the appraisal and follow-up of birdshot chorioretinopathy. Arch Ophthalmol 116(10):1389-1391

5. Thorne JE, Jabs DA, Kedhar SR, Peters GB, Dunn JP (2008) Loss of visual field among patients with birdshot chorioretinopathy. Am J Ophthalmol 145(1):23-28, Epub 2007 Nov 12

6. Gordon LK, Goldhardt R, Holland GN, Yu F, Levinson RD (2006) Standardized visual field assessment for patients with birdshot chorioretinopathy. Ocul Immunol Inflamm 14(6):325-332 
7. Levinson RD, Brezin A, Rothova A, Accorinti M, Holland GN (2006) Research criteria for the diagnosis of birdshot chorioretinopathy: results of an international consensus conference. Am J Ophthalmol 141(1):185-187

8. Herbort CP, LeHoang P, Guex-Crosier Y (1998) Schematic interpretation of indocyanine green angiography in posterior uveitis using a standard angiographic protocol. Ophthalmology 105(3):432-440

9. Monnet D, Brézin AP, Holland GN, Yu F, Mahr A, Gordon LK, Levinson RD (2006) Longitudinal cohort study of patients with birdshot chorioretinopathy. I. Baseline clinical characteristics. Am J Ophthalmol 141(1):135-142

10. Rothova A, Suttorp-van Schulten MS, Frits Treffers W, Kijlstra A (1996) Causes and frequency of blindness in patients with intraocular inflammatory disease. Br J Ophthalmol 80(4):332-336

11. Rothova A, Berendschot TT, Probst K, van Kooij B, Baarsma GS (2004) Birdshot chorioretinopathy: long-term manifestations and visual prognosis. Ophthalmology 111(5):954-959

12. Gass JD (1981) Vitiliginous chorioretinitis. Arch Ophthalmol 99 (10):1778-1787

13. Guex-Crosier Y, Herbort CP (1997) Prolonged retinal arteriovenous circulation time by fluorescein but not by indocyanine green angiography in birdshot chorioretinopathy. Ocul Immunol Inflamm 5(3):203-206

14. Bouchenaki N, Herbort CP (2001) The contribution of indocyanine green angiography to the appraisal and management of VogtKoyanagi-Harada disease. Ophthalmology 108(108):54-64

15. Herbort CP, Mantovani A, Bouchenaki N (2007) Indocyanine green angiography in Vogt-Koyanagi-Harada disease: angiographic signs and utility in patient follow-up. Int Ophthalmol 27:173-182

16. Kawaguchi T, Horie S, Bouchenaki N, Ohno-Matsui K, Mochizuki M, Herbort CP (2010) Suboptimal therapy controls clinically apparent disease but not subclinical progression of Vogt-KoyanagiHarada disease. Int Ophthalmol 30:41-50

17. Wolfensberger TJ, Herbort CP (1999) Indocyanine green angiographic features in ocular sarcoidosis. Ophthalmology 106:285-289
18. Wolfensberger TJ, Piguet B, Herbort CP (1999) Indocyanine green angiographic (ICGA) features in tuberculous chorioretinitis. Am J Ophthalmol 127:350-353

19. Ryan S, Maumenee AE (1980) Birdshot retinochoroidopathy. Am J Ophthalmol 89:31-45

20. Cimino L, Auer C, Herbort CP (2000) Sensitivity of indocyanine green angiography for the follow-up of active inflammatory choriocapillaropathies. Ocul Immunol Inflamm 8:275-283

21. Herbort CP (1998) Posterior uveitis: new insights provided by indocyanine green angiography. Eye 12:757-759

22. Fardeau C, Herbort CP, Kullmann N, Quentel G, LeHoang P (1999) Indocyanine green angiography in Birdshot chorioretinopathy. Ophthalmology 106:1928-1934

23. Herbort CP, Bodaghi B, LeHoang P (2001) Angiographie au vert d'indocyanine au cours des maladies oculaires inflammatoires: principes, interprétation schématique, sémiologie et intérêt clinique. J Fr Ophtalmol 24:423-447

24. Thorne JE, Jabs DA, Peters GB, Hair D, Dunn JP, Kempen JH (2005) Birdshot retinochoroidopathy: ocular complications and visual impairment. Am J Ophthalmol 140(1):45-51

25. Cimino L, Tran VT, Herbort CP (2002) Importance of visual field testing in the functional evaluation and follow-up of birdshot chorioretinopathy. Ophthalmic Res 34(S1):141

26. Lim L, Harper A, Guymer R (2006) Choroidal lesions preceding symptom onset in birdshot chorioretinopathy. Arch Ophthalmol 124(7):1057-1058

27. Machida S, Tanaka M, Murai K, Takahashi T, Tazawa Y (2004) Choroidal circulatory disturbance in ocular sarcoidosis without the appearance of retinal lesions or loss of visual function. Jpn J Ophthalmol 48(4):392-396

28. Howe LJ, Stanford MR, Graham EM, Marshall J (1997) Choroidal abnormalities in birdshot chorioretinopathy: an indocyanine green angiography study. Eye 11(4):554-559

29. Gaudio PA, Kaye DB, Crawford JB (2002) Histopathology of birdshot retinochoroidopathy. Br J Ophthalmol 86(12):1439-1441 